\title{
AIAA 2001-0560 \\ Determining Aerodynamic Loads Based on Optical Deformation Measurements
}

Tianshu Liu, D. A. Barrows, A. W. Burner and R. D. Rhew

NASA Langley Research Center

Hampton, VA 23681-2199

\section{9th AIAA Aerospace Sciences Meeting \& Exhibit 8-11 January 2001 / Reno, NV}

For permission to copy or republish. contact the American Institute of Aeronautics and Astronautics 1801 Alexander Bell Drive, Suite 500, Reston, VA 20191 


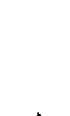




\title{
Determining Aerodynamic Loads Based on Optical Deformation Measurements
}

\author{
Tianshu Liu ${ }^{\dagger}$, D. A. Barrows ${ }^{*}$, A. W. Burner and R. D. Rhew ${ }^{\dagger}$ \\ NASA Langley Research Center \\ Hampton, VA 23681-2199
}

\begin{abstract}
This paper describes a videogrammetric technique for determining aerodynamic loads based on optical elastic deformation measurements. The data reduction methods are developed to extract the normal force and pitching moment from heam deformation data. The axial force is ohtained by measuring the axial translational motion of a movable shaft in a spring/bearing device. Proof-ol-concept calibration experiments are conducted to assess the accuracy of this optical technique.
\end{abstract}

\section{Introduction}

Internal strain gauge balances have been used for years as a standard technique for measuring the integrated aerodynamic forces and moments in wind tunnels. A variety of internal strain gauge balances have been developed and the technical aspects of various balances have been studied in detail $11 \mid$. Generally speaking, the structure of an internal strain gauge balance is complicated and the cost of fabrication is high. This paper presents an exploratory study for remotely measuring aerodynamic loads using a videogrammetic system. Unlike strain gauges, this method optically measures heam deformation to determine the normal force and pitching moment. The axial force is obtained by measuring the translational motion of a movable shaft in a spring/hearing device. Mathematical models for data reduction are developed to extract the acrodynamic forces and moments from deformation data. Uncertainty analysis is given to evaluate the contributions from the elemental error sources and correlation terms. At this stage, the normal force, pitching moment, and axial force are the primary quantities to he delermined. In principle, the side force, rolling moment, and yawing moment can be determined in a similar manner. Proof-of-concept laboratory experiments have been conducted to validate the proposed methodology of measuring the aerodynamic loads. Potentially, the optical method can be used as an alternative to strain gauge

\footnotetext{
+ Research Scientist. Model Systems Branch. Member AlAA

$\therefore$ Research Scientisi, Instrumentation Systems Development Branch. Senior Member AIAA

* Engineer. Instrumentation Systems Development Branch

Copyright $\odot 2001$ by the American Institute of Aeronautics and Astronautics, Inc. No copyright is asserted in the United States under Title 17, U.S. Code. The U.S. Government has a royalty-free license to exercise all rights under the copyright claimed herein for Governmental purposes All other rights are reserved by the copyright owner.
}

balances. In addition, the technique described in this paper can be easily integrated with the optical model attitude measurement techniques $[2,3]$.

\section{Cantilever Beam Deformation}

Deformation of a cantilever beam is utilized to calculate the normal fores and pitching moment. Consider a cantilever beam bent by a force $F$ and a moment $M$ applied at the end, as shown in Fig. 1. According to the engineering beam theory in which the deformation due to shear strain is assumed to be negligible [4]. the normal coordinate $v$ of the beam is described by

$$
E I(x) y_{x y}=-F(L-x)-M \text {. }
$$

where $v_{A x}$ is the second derivative with respect to $x, M$ is the local bending moment, $E$ is the modulus of elasticity. and $I(x)$ is the moment of inertia. The boundary conditions are $v_{1}(x=0)=v_{x}$ and $v(x=0)=v_{0}$. The solution for the displacement $\delta v=v-v_{0}-v_{3} x$ is

$$
\delta(x)=-E^{-1}(F L+M) Q_{1}(x)+E^{-1} F Q_{2}(x) .
$$

where

$$
\begin{aligned}
& \left.Q_{1}(x)=\int_{0}^{1} d x^{\prime} \int_{0}^{1} I I\left(x^{\prime \prime}\right)\right]^{-1} d x^{\prime \prime} . \\
& Q_{2}(x)=\left.\int_{0}^{1} d x^{\prime} \int_{0}^{x^{\prime}} I I\left(x^{\prime \prime}\right)\right|^{-1} x^{\prime \prime} d x^{\prime \prime} .
\end{aligned}
$$

For a rectangular cross-section beam, the moment of inertia is $I=w h^{3} / I 2$, where $w$ is the width and $h$ is the height of the cross-section. For a circular cross-section beam. the moment of inertia is $I=\pi R^{+} / 4$, where $R$ is the radius of the circular cross-section. When a beam has a constant cross-section and the moment of inertia is constant, the expression (2) hecomes

$$
\dot{\delta} v(x)=-(E I)^{-1}(F L+M) x^{2} / 2+(E I)^{-1} F x^{2} / 6 .
$$

Therefore, the local displacement $\delta v$ and the slope change

$$
\begin{aligned}
\delta v_{x}=v_{x}-v_{x} \text { are } & \left(\begin{array}{l}
\delta v \\
\delta v_{x}
\end{array}\right)=\left(\begin{array}{ll}
x^{3} / 6 E l & -x^{2} / 2 E I \\
x^{2} / 2 E I & -x / E I
\end{array}\right)\left(\begin{array}{l}
F \\
M
\end{array}\right),
\end{aligned}
$$

where $M_{0}=F L+M$ is the moment with respect to a moment center $c$. Eq. (4) gives a linear relation between the deformation $(\delta v, \delta v$, and the force and moment 
$\left(F, M_{c}\right)$. When the deformation due to shear strain is taken into account, a more complete analysis of a rectangular cantilever beam gives a non-linear relation [5]

$$
\begin{aligned}
& \delta v^{\prime}=\left(x^{3} / 6 E I\right) F-(x / 2 E l) F L^{2}+(1 / 3 E I) F L^{3} . \\
& \delta v_{x}=\left(x^{2} / 2 E I\right) F-(1 / 2 E I) F L^{2}
\end{aligned}
$$

A generalized relation can be written as

$$
\delta v=\sum_{i=0} C_{1 j} F L^{j} \text { and } \delta v_{i}=\sum_{j=0} C_{2 j} F L^{j} .
$$

where $C_{i j}$ are the coefficients to be determined in calibration tests using the least-squares method. These theoretical results provide basic models for data reduction to recover the force and moment. Generally, given the force and moment, the theoretical relations describe beam deformation profiles well. However, inversion to the force and moment from deformation measurements is a "stiff" problem that is very sensitive to small errors. In practical data-reduction procedures, these theoretical results will be combined with empiricism to deal with imperfections in the real measurements.

\section{Data Reduction Methods}

\subsection{Method based on local displacement and slope}

Eqs. (4) and (5) indicate that the force $F$ and moment $M$. depend on the local displacement $\delta v$ and the change of slope $\delta v_{x}$. Therefore, $\left(F, M_{i}\right)$ can be determined from measurements of $\left(\delta v_{,} \delta v_{x}\right)$. Here, the pitching moment $M_{1}$ is defined as $M_{c}=F\left(L-L_{c}\right)$, where $L$ is the distance between the force (load) location and the beam support and $L_{t}$ is the distance between the moment center $c$ and the support. The local displacement and the change of slope are

$$
\begin{aligned}
& \delta v=v(x)-v\left(x_{r f f}\right)-v_{x}\left(x_{r f f}\right) x_{r e t} \\
& \delta v_{x}=v_{x}(x)-v_{x}\left(x_{r e f}\right),
\end{aligned}
$$

where $x_{r e f}$ is the reference location where deformation is small such as the position near the support. Instead of directly using the definition (7), the practical procedures for calculating $\left(\delta v_{1} \delta v_{x}\right)$ are based on the affine transformation because of its rohustness.

The quantities $\delta v$ and $\delta v$, are calculated from the optically measured coordinates of high-contrast targets on the beam. Figure 2 shows a typical layout of targets on a beam (sting) assembled to a wind tunnel model. A row of targets, denoted by $B$ in Fig. 2, is placed in parallel to the beam centerline near the wind tunnel model. Another row of targets, reference targets denoted by $\mathrm{A}$, is placed near the support of the beam. When the beam is deflected by aerodynamic loads, not only the targets at B move, but also the reference targets at $\mathrm{A}$ may move slightly since the support is not absolutely rigid. Thus, the total movement of target row $B$ contains both the relative deformation at $B$ with respect to $A$ and the local movement at $A$. The local deformation at B, which is more sensitive to loads, can be obtained by removing the local movement at $A$ from the total movement at $B$.

In order to correct the movement of the reference target row $A$ due to loads, the load-off (or wind-oft) position of the target row $\mathrm{A}$ is used as a baseline position. Assuning that the local movement of the reference target row A can be approximated as rigid-body motion, one obtains the affine transformation between the load-off coordinate $(\tilde{X}, \tilde{Z})$ and load-on coordinate $(X, Z)$ for the reference target row $\mathrm{A}$

$$
\left(\begin{array}{c}
\tilde{X} \\
\tilde{Z}
\end{array}\right)=R_{A}\left(\begin{array}{l}
X \\
Z
\end{array}\right)+\left(\begin{array}{l}
T_{A A} \\
T_{Z A}
\end{array}\right),
$$

where $R_{A}$ is the rotational matrix

$$
R_{A}=\left(\begin{array}{cc}
\cos \theta_{A} & \sin \theta_{A} \\
-\sin \theta_{A} & \cos \theta_{A}
\end{array}\right),
$$

$\theta_{A}$ is a rotational angle at $A$, and $T_{x A}$ and $T_{F A}$ are the translations at $\mathrm{A}$. The coordinate system $(X, Y, Z)$ is a standard wind-tunnel coordinate system in which $X$ is in the free-stream flow direction, $Y$ is in the spanwise direction of a wind-tunnel model at zero angle-of-attack, and $Z$ is in the vertical direction. The beam deformation is in the $(X, Z)$ plane. The rotational angle $\theta_{A}$ and the translations ( $T_{x A}$, $T_{i A}$ ) can be determined from the measured load-off and load-on coordinates of target row $A$ by using the leastsquares method.

Applying the affine transformation (8) to the load-on coordinates of the target row $\mathrm{B}$, we are able to eliminate the effect of the local movement at $A$. We denote the transformed load-on coordinates of target row $B$ by $\left(\tilde{X}_{B m}, \tilde{Z}_{B m}\right)$ and call them the re-aligned load-on coordinates of target row $B$ relative to the reference luadoff target row $\mathrm{A}$. The re-aligned load-on coordinates $\left(\tilde{X}_{B e n}, \tilde{Z}_{B m}\right)$ of the target row $B$ are related to the corresponding load-off coordinates $\left(\tilde{X}_{B u f f}, \tilde{Z}_{B u f f}\right)$ by the affine transformation

$$
\left(\begin{array}{l}
\tilde{X}_{B u m} \\
\tilde{Z}_{B u n}
\end{array}\right)=R_{B}\left(\begin{array}{l}
\tilde{X}_{B u f t} \\
\tilde{Z}_{B u t f}
\end{array}\right)+\left(\begin{array}{l}
T_{x B} \\
T_{z B}
\end{array}\right),
$$

where $R_{B}$ is the rotational matrix

$$
R_{B}=\left(\begin{array}{cc}
\cos \theta_{B} & \sin \theta_{B} \\
-\sin \theta_{B} & \cos \theta_{B}
\end{array}\right)
$$

The change of the bending angle at $B$ is $\theta_{B}$ and $\Delta \boldsymbol{r}_{B}=\left(T_{x B}, T_{z B}\right)$ is the displacement vector at $\mathrm{B}$. The 
values of $\theta_{B}$ and $\Delta \boldsymbol{r}_{B}=\left(T_{A B}, T_{Z B}\right)$ can be determined by using the least-squares method and they are the average quantities of local deformation for target row B. Therefore. the change of the slope at $\mathrm{B}$ due to loads is simply

$$
\delta_{v_{i}}=\tan \left(\theta_{B}\right) \text {. }
$$

The normal displacement $\delta v$ at B due to loads is

$$
\delta v^{\prime}=n_{B} \cdot \Delta r_{B},
$$

where $n_{R}$ is the unit vector normal to the beam axis at B.

In reality, the relationship between $\left(F, M_{r}\right)$ and $\left(\delta v, \delta v_{1}\right)$ is more complicated than theoretical prediction by the engineering beam theory. The relations for calculating $(F, M$,$) are generally expressed as$

$$
F=f_{1}\left(\delta v, \delta v_{x}\right) \text { and } M_{i}=f_{2}\left(\delta v, \delta v_{1}\right) \text {. }
$$

In practice. we do not tend to globally fit the whole set of calibration data to obtain the complete functional relations. Instead, for a given data point $\left(\delta v_{,} \delta v_{y}\right)$, we use a local 2nd-order polynomial to interpolate a group of neighboring calibration data points, that is,

$$
F=B_{1} \delta \text { and } M_{i}=B_{2} \delta \text {. }
$$

where $\delta$ is the deformation vector defined as

$$
\delta=\left(\delta v, \delta v_{,},(\delta v)^{2}, \delta v \delta v_{1},(\delta v,)^{2}\right)^{T}
$$

and $\boldsymbol{B}_{1}$ and $\boldsymbol{B}_{2}$ are the coefficients determined by calibration.

A simple model approach can be also used to recover $\left(F, M_{c}\right)$ from given $\left(\delta v, \delta v_{x}\right)$. It is found that for a suitably chosen moment center $L_{c}$, the moment $M_{i}=F\left(L-L_{i}\right)$ is simply a linear function of either $\delta v$ or $\delta v_{x}$. This is a reduced case in which $\delta v$ is proportional to $\delta v_{1}$. In this case, the moment $M_{\text {, is given }}$ by a simple proportional relation

$$
M_{\mathrm{i}}=F\left(L-L_{\mathrm{t}}\right)=\alpha(\delta v) \text {, }
$$

where $\alpha$ is a proportional constant determined by calibration. The best moment center $x=L_{t}$, to achieve the linearity given by $\mathrm{Eq}$. (16) can be determined by an optimization scheme. In addition, an empirical relation between $F$ and $\delta v$ is

$$
\delta_{v}=\left(\beta_{i}+\beta_{1}\left(L-L_{i}\right)+\beta_{2}\left(L-L_{i}\right)^{2} / F .\right.
$$

where $\beta_{0}, \beta_{1}$, and $\beta_{2}$ are constants determined in calibration. Eliminating ( $L-L_{c}$ ) in Eqs. (16) and (17). one obtains a 2 nd-order algebraic equation for $F$

$$
\beta_{0} F^{2}+\left(\beta_{1} M_{,}-\delta v\right) F+\beta_{2} M_{1}^{2}=0 \text {. }
$$

In principle. $F$ and $M_{1}$ can be obtained from Eqs. (16) and (18) for a given $\delta v$. However, Eq. (18) has two real roots that are often close each other. It is not easy to choose the correct solution without a good initial guess of $F$. This is a shortcoming of the simple model approach.

\subsection{Method based on global beam deformation profile}

From Eq. (3), one knows that the displacement $\delta$ : along the beam axis can be described by the theoretical relation

$$
\delta v(x)=a x^{2}+b x^{3}
$$

where $x$ is the coordinate along the beam axis $\delta y$ is obtained using Eq. (13), and the coefficients $a$ and $b$ are related to $F$ and $M_{1}$. However, the relation between $(a, b)$ and $\left(F, M_{1}\right)$ is not as simple as that given by Eq. (3). The empirical relations are symbolically expressed as

$$
F=f_{1}(a, b) \text { and } M_{2}=f_{2}(a, b) \text {. }
$$

For a given data point $(a, b)$. a local 2 nd-order polynomial fit to a group of neighboring calibration data points is used to recover $(F, M$,$) .$

As an alternative, we also use a simple model approach to calculate $\left(F, M_{4}\right)$. The empirical lunctional relations are

$$
\begin{aligned}
& a=\mid a_{0}+a_{1}\left(L-L_{1}\right)+a_{2}\left(L-L_{i}\right)^{2} / F \\
& b=\left(b_{0}+b_{1}\left(L-L_{1}\right)+b_{2}\left(L-L_{i}\right)^{2} \mid F .\right.
\end{aligned}
$$

where $\left(a_{0}, a_{1}, a_{2}\right)$ and $\left(b_{0}, b_{1}, b_{2}\right)$ are determined in calibration. The solution to Eq. (21) is

$$
\begin{aligned}
& \left.L-L_{i}=\left(-c_{1} \pm \sqrt{\left.c_{1}^{2}-4 c_{2} c_{1}\right)}\right) / 2 c_{2}\right) \\
& \left.F=a / / a_{0}+a_{1}\left(L-L_{1}\right)+a_{2}\left(L-L_{1}\right)^{2}\right) .
\end{aligned}
$$

where $\quad c_{0}=b_{0} a / b-a_{0}, \quad c_{1}=b_{1} a / b-a_{1}$. and $c_{2}=b_{2} a / b-a_{2}$.

\subsection{Uncertainty}

For uncertainty analysis, consider the general functional relations between $(F, M$,$) and \left(\delta v, \delta_{v},\right)$

$$
F=f_{1}\left(\delta v, \delta v_{x}, p\right) \text { and } M_{1}=f_{2}\left(\delta v_{,} \delta v_{x}, q\right) \text {, }
$$

where $\boldsymbol{p}=\left|p_{1}, p_{2}, \cdots p_{N}\right|$ and $\boldsymbol{q}=\left|q_{1}, q_{2}, \cdots q_{N}\right|$ are the parameters characterizing the functional relations. Standard uncertainty analysis $[6]$ gives the error propagation equations for the relative variances of $F$ and $M$,

$$
\begin{aligned}
& \frac{\operatorname{var}(F)}{F^{2}}=\sum_{i=1}^{N} S_{i p_{i}}^{2} \frac{\operatorname{var}\left(p_{i}\right)}{p_{i}^{2}}+S_{i \delta v^{\prime}} \frac{\operatorname{var}(\delta v)}{(\delta v)^{2}}+S_{i \delta v^{\prime}}^{2} \frac{\operatorname{var}\left(\delta v_{i}\right)}{\left(\delta v_{i}\right)^{2}} \\
& +S_{1 \delta v}, S_{1 \delta v,} R\left(\delta v, \delta v, \frac{[\operatorname{var}(\delta v) \operatorname{var}(\delta v,)]^{1 / 2}}{\delta v \delta v}\right. \\
& \frac{\operatorname{var}\left(M_{1}\right)}{M_{i}^{2}}=\sum_{i=1}^{N} S_{2 q_{i}}^{2} \frac{\operatorname{var}\left(q_{i}\right)}{q_{i}^{2}}+S_{2}^{2} \delta \cdot \frac{\operatorname{var}(\delta v)}{(\delta v)^{2}}+S_{2}^{2} \delta v^{2} \frac{\operatorname{var}\left(\delta v_{1}\right)}{\left(\delta v_{1}\right)^{2}} \\
& +S_{2 \delta v}, S_{2 \delta v}, R\left(\delta v, \delta v_{1}\right) \frac{\operatorname{lrar}\left(\delta v \operatorname{mar}(\delta v, 1)^{1 / 2}\right.}{\delta i \delta v}
\end{aligned}
$$

where the sensitivity coefticients are

$$
S_{i p_{i}}=\left(p_{i} / F\right)\left(\partial F / \partial p_{i}\right) \text {. }
$$




$$
\begin{aligned}
& S_{2 q_{i}}=\left(q_{i} / M_{c}\right)\left(\partial M_{i} / \partial q_{i}\right), \\
& S_{/ \delta v}=(\delta v / F)(\partial F / \partial \delta v), \\
& S_{/ \delta v^{\prime}}=\left(\delta v_{x} / F\right)\left(\partial F / \partial \delta v_{x}\right), \\
& S_{2 \delta v^{\prime}}=\left(\delta v / M_{c}\right)\left(\partial M_{c} / \partial \delta v\right), \text { and } \\
& S_{2 \delta v_{1}}=\left(\delta v_{x} / M_{,}\right)\left(\partial M_{c} / \partial \delta v_{x}\right) .
\end{aligned}
$$

The correlation coefficient $R\left(\zeta_{i}, \zeta_{j}\right)$ between the variables $\zeta_{i}$ and $\zeta_{i}$ is defined as $R\left(\zeta_{i}, \zeta_{j}\right)=\operatorname{cov}\left(\zeta_{i} \zeta_{j}\right) / /\left.\operatorname{var}\left(\zeta_{i}\right) \operatorname{var}\left(\zeta_{j}\right)\right|^{1 / 2}$, and the variance and covariance are $\operatorname{var}\left(\zeta_{i}\right)=\left\langle\Delta \zeta_{i}^{2}\right\rangle$ and $\operatorname{cov}\left(\zeta_{i} \zeta_{j}\right)=\left\langle\Delta \zeta_{i} \Delta \zeta_{j}\right\rangle$, where the notation $<>$ denotes the statistical assemble average and $\Delta \zeta_{i}=\zeta_{i}-\left\langle\zeta_{i}\right\rangle$ is the variation. There are two types of contributions to the total uncertainties in $F$ and $M_{c}$. The summation terms in the right-hand side of Eqs. (24) and (25) are the uncertainties in fitting the coefficients $p$ and $q$ in the data-reduction models. The other three terms in the right-hand side are the uncertainties in measurements of $\left(\delta v, \delta v_{x}\right)$. The uncertainties in measurements of $\left(\delta v^{\prime} \delta v_{x}\right)$ are determined by the accuracy of the camera calibration (about 1:60000 for the videogrammetric system used). In addition. an important error source that is not included in Eqs. (24) and (25) is a bias error in the data-reduction mathematical model itself since the model may fail to describe correctly actual measurement processes.

The last terms in the right-hand side of Eqs. (24) and (25) are the correlation terms that are more intriguing since they could be positive or negative. The total uncertainties decrease when the correlation terms are negative and otherwise the total uncertainties increase. Based on the linear theoretical relation (4), the correlation between $\delta v$ and $\delta v_{x}$ can be calculated, that is,

$$
\begin{aligned}
& <\Delta(\delta v) \Delta\left(\delta v_{k}\right)>= \\
& \left.\quad \frac{x^{3}<(\Delta F)^{2}>}{I 2(E l)^{2}} \mid x-2\left(L-L_{c}\right)\right) /\left(x-3\left(L-L_{c}\right)\right)^{\prime}
\end{aligned}
$$

The correlation terms in Eqs. (23) and (24) are

$$
\begin{aligned}
& S_{/ \delta} S_{/ \delta v_{1}} \frac{\left\langle\Delta(\delta v) \Delta\left(\delta v_{x}\right)>\right.}{\delta v \delta v_{1}}= \\
& \frac{<(\Delta F)^{2}>}{x^{2} F^{2}}\left(x-2\left(L-L_{c}\right)\right)\left(x-3\left(L-L_{c}\right)\right) \\
& S_{2 \delta} S_{2 \delta v_{1}} \frac{\left\langle\Delta(\delta v) \Delta\left(\delta v_{x}\right)\right\rangle}{\delta v \delta v_{x}}= \\
& \frac{<(\Delta F)^{2}>}{6 M_{c}^{2}}\left[x-2\left(L-L_{c}\right)\right)\left(x-3\left(L-L_{c}\right)\right)
\end{aligned}
$$

The correlation terms are negative for $2\left(L-L_{c}\right)<x<3\left(L-L_{c}\right)$ and $0<L_{c}<L$. Furthermore, at a fixed target location $x=r L \quad(r \leq l)$, the correlation terms are negative when the moment center $x=L_{c}$ is in $(2-r) L / 2<L_{c}<(3-r) L / 3$. This analysis indicates that the moment center can be suitably selected to reduce the total uncertainties in measurements.

\section{Videogrammetric System}

In this study, elastic deformation of a beam is measured using a videogrammetric system. Based on the principles of close-range photogrammetry, the videogrammetric system measures the coordinates of targets distributed along the beam from target centroids in digital images. Deformation of the beam is then calculated from the measured target coordinates. Figure 3 shows a schematic of a two-camera videogrammetric system for deformation measurements. Basic hardware of the system consists of two Hitachi KP-F1 CCD cameras with $15-\mathrm{mm}$ lenses, a Dell PC with a Matrox Pulsar frame grabber board, light sources and retro-reflecting targets on the beam. Software includes programs for image acquisition, target-tracking/centroid calculation, and camera calibration. This system is able to provide three-dimensional coordinates $(X, Y, Z)$ in almost real time. The accuracy of the videogrammetric system used for this work is typically 1:60000. After the coordinates of the targets on the beam are obtained, the deformation ( $\delta v, \delta v_{x}$ ) can be calculated. The data-reduction programs for calculating the deformation, force and moment are written in Matlab. The technical aspects of the videogrammetric system have been described in detail by Liu et al. [7,8]. Comprehensive reviews of application of videogrammetry to wind tunnel testing have been given by Burner \& Liu [2], and Liu et al. [8].

\section{Experimental Results \\ 5.1. Simple beams}

Figure 4 shows a standard balance calibration apparatus and a two-camera videogrammetric system. Three simple beams have been calibrated to examine the proposed methodology of recovering the normal force and pitching moment (see Fig. 5). One is a brass beam with a $0.75 \mathrm{in} \times 0.75 \mathrm{in}$ square cross-section and the rigidity of $E I=350 \times 10^{3}{\mathrm{lb}-\mathrm{in}^{2}}^{2}$. A more flexible stainless steel beam is actually a flat plate that has a rectangular cross-section of $1.5 \mathrm{in} \times 0.25 \mathrm{in}$ and the rigidity of $E I=47 \times 10^{3} \mathrm{lb}^{\mathrm{bin}}{ }^{2}$. A more rigid stainless steel beam was also used, which has a circular cross-section with a diameter $0.75 \mathrm{in}$ and the rigidity of $E I=442 \times 10^{3} \quad \mathrm{lb}-\mathrm{in}^{2}$. A number of retroreflecting targets used for deformation measurements are placed along the centerline of a beam.

Figures 6 and 7 show the local displacement and slope change $\left(\delta v, \delta v_{1}\right)$ as a function of the normal force for the brass beam at four different loading positions. It is found 
that the dependence of both the local displacement and slope change on the pitching moment is linear when the moment center is suitably chosen. The linear relations are clearly shown in Fig. 8 for the brass beam, where the moment center determined by an optimization scheme is located at $L_{\mathrm{c}}=9.03 \mathrm{in}$. This linearity is utilized in the simple model method for data reduction. Figure 9 shows the errors in measurements of the normal force and pitching moment by using the method hased on local displacement and slope change for the brass beam. The local 2 nd-order polynomial and the simple model methods are used for data interpolation. For the soft and stiff stainless steel heams, Figure 10) shows the measurement errors in the normal force and pitching moment. Typically, the relative errors in the normal force and pitching moment are within $\pm 10 \%$ and $\pm 5 \%$, respectively.

The method hased on global deformation profile is used to recover the normal force and pitching moment for the brass beam. Figure 11 shows measured deformation profiles of the brass heam for different loads at the loading position $L=28.069$ in. These measured data can be well fit by the theoretical solution $\delta v(x)=a x^{2}+b x^{3}$. The errors in measurements of the normal force and pitching moment are shown in Fig. 12, where the local 2 nd-order polynomial and simple model methods are used for data interpolation. Compared to the method based on the local displacement and slope change, the method based on the global deformation profile gives smaller relative errors in the normal force (within $\pm 5 \%$ ) and pitching moment (within $\pm 3 \%$ ) for the brass beam.

\subsection{Sting-model combination}

A steel sting-model combination used in the Unitary Tunnel at NASA Langley was calibrated, as shown in Fig. 13. The sting has a tapering circular cross-section with a linearly decreasing radius from $0.5975 \mathrm{in}$. to $0.197 \mathrm{in}$. Accordingly, the rigidity decreases from $2800 \times 100^{3}$ to $38 \times 10^{-} \mathrm{lb}-\mathrm{in}^{2}$ and the mean rigidity of the sting is $930 \times 10^{.} \quad \mathrm{lb}^{2} \mathrm{in}^{2}$. In calibration tests, the maximum displacement of the sting is about 0.03 in and the maximum change of the local bending angle is about 0.23 degrees. Two strain gauge bridges were also installed on the sting for measurement of the normal force and pitching moment, allowing a direct comparison between the strain gauge method and the optical method.

During calibration tests, a number of dead weights from $2 \mathrm{lb}$ to $10 \mathrm{lb}$ were loaded at three different locations on the model to generate the required forces and moments. Three retro-reflecting targets were placed near the model for measuring local deformation and four other targets were placed near the support as a reference. The local deformation quantities $(\delta v, \delta v$, ) were measured using the videogrammetric system. The method based on the local displacement and slope change was used to determine the normal force and pitching moment. Figure 14 shows the measurement errors in the normal force and pitching moment for this steel sting-model combination. The results indicate that the optical method based on remote deformation measurement is less accurate than the more sensitive strain gauges. The relative errors in the normal force and pitching moment obtained by the optical method are about $\pm 5 \%$ in comparison with $\pm 2 \%$ given by the strain gauges.

\subsection{Three-force beam}

In order to measure the axial force along with the normal force and pitching moment, a three-force beam has been designed and fabricated. As shown in Fig. 15, the three-force beam consists of a simple beam and a spring/bearing device that only allows translational motion along the beam axis. The structure of the spring/bearing device is shown in Fig. 16. A steel axial load shaft (made of medium-alloy A-2 stecl) is a moving rod that is constrained by a linear bearing mounted inside the hardware assembly. The rod is hardened and has a ground surface finish of $0.625(+0.00(0) 2 /-0.00(0))$ inches on the diameter. This tight tolerance allows a very close interface fit with the bearing so all lateral movement is minimized. Another main component of the device is the spring that balances the applied axial force. Ranges of the springs with different spring constants are available and can be selected to meet the requirements of the axial force calibration. The three-force beam attaches to the extended end of the 0.625 diameter stainless steel beam. The device housing has an outside diameter of 2.38 inches and is 6.0 inches long with an extended length of the entire heam assembly of 28.25 inches in the balance calibration apparatus. As shown in Fig. 15, a number of retro-reflecting targets are placed on the simple beam for measuring beam deformation. Four targets are placed on the spring/hearing device as a reference and the other four targets are placed on the movable shaft for measuring the axial translational motion relative to the reference targets. The average spacing hetween retro-reflective targets is approximately 0.65 inches.

The normal force and pitching moment are obtained by using the same methods as previously described. The measurement errors in the normal force and pitching moment are shown in Fig. 17, indicating $\pm 4 \%$ errors for the normal force and $\pm 3 \%$ errors for the pitching moment. The axial force $F_{a x}$ is related to the axial displacement $\delta x$ by a linear relation

$$
F_{a x}-F_{a x}=k \delta x
$$

where $k$ is an effective spring coefficient and $F_{\text {axo }}$ is the force at $\delta x=0$. When the normal force $F_{N}$ and pitching moment $M_{1}$ exist, $k$ and $F_{a x}$ are not constants and they are weakly dependent on $F_{N}$ and $M_{1}$, due to the presence of friction in the hearing. Experiments show that $k$ and $F_{a x}$ are mainly related to the loading position $M_{i} / F_{N}$ 
over a certain range of the normal force. Thus, an empirical calibration relation for the axial force is

$$
F_{a x}=k\left(M_{c} / F_{N}\right) \delta x+F_{u x o}\left(M_{,} / F_{N}\right),
$$

where $k\left(M_{c} / F_{N}\right)$ and $F_{a x o}\left(M_{c} / F_{N}\right)$ are empirically expressed by polynomials. Figure 18 shows the linear relation between the axial force and the measured axial displacement. As shown in Fig. 19, the measurement errors in the axial force obtained by using Eq. (29) are within $\pm 8 \%$.

\section{Conclusions}

The methodology of optically measuring aerodynamic loads is developed based on beam deformation theory. A two-camera videogrammetric system is used for optical deformation measurements. The data reduction models for extracting the normal force and pitching moment utilize either the local displacement and slope change or the global beam deformation profile. The interpolation methods for calibration data include the local 2 nd-order polynomial fit and the simple model approach. On the other hand, the axial force is obtained by measuring the translational motion of a movable shaft in a spring/bearing device. In order to validate the proposed technique, three simple metallic beams with different rigidities have been tested to recover the normal force and pitching moment. Typically, the relative errors in the normal force and pitching moment are, respectively, from $\pm 5 \%$ to $\pm 10 \%$ and $\pm 2 \%$ to $\pm 5 \%$ for the three beams. A steel sting-model combination was also calibrated for a direct comparison between the strain gauge method and the optical method. The loads obtained by the optical method have larger errors than the strain gauges. To determine the axial force, a three-force beam that allows the axial translational motion has been designed and fabricated. The measurement errors in the axial force are within $\pm 8 \%$. At this stage, the optical method is less accurate than the well-developed strain gauge method. Nevertheless, the optical method has the capability of remote non-contact measurement and it can be readily integrated with the optical methods of model attitude measurement. The optical method described in this paper can be considered as an alternative in certain cases where the strain gauge method cannot be easily applied. Further research effort will be made to improve the accuracy of the optical method in measuring aerodynamic loads and apply the technique to wind tunnel testing.

\section{References:}

[1] Tripp, J. S. and Tcheng. P., Proceeding of First International Symposium on Strain Gauge Balances, NASA Langley Research Center, Hampton, Virginia, March 1999.

[2] Burner, A. W. and Liu, T., Videogrammetric model deformation measurement technique, Journal of Aircraft, 2000 (in press).
[3] Burner, A. W., Model deformation measurements at NASA Langley Research Center, AGARD Conference Proceedings CP-601: Advanced Aerodynamic Measurement Technology, presented at the $81 \mathrm{st}$ Meeting and Symposium of the Fluid Dynamics Panel, Seattle, WA, September, 1997, pp. 34-1-34-9.

[4] Bisplinghoff, R., Mar, J. W., and Pian, T. H. H., Statics of Deformable Solids, Addison-Wesley, New York, 1965.

[5] Timoshenko, S. and Goodier, J. N., Theory of Elasticity, McGraw-Hill Book Company, New York, 1970.

[6] Bevington, P. R. and Robinson, D. K., Data reduction and error analysis for the physical sciences (Second Edition), McGraw-Hill, Inc., New York, 1992.

[7] Liu, T., Radeztsky, R., Garg, S., and Cattafesta, L., A videogrammetric model deformation system and its integration with pressure paint, AIAA Paper 99-0568, 1999.

[8] Liu, T., Cattafesta, L., Radeztsky, R. and Burner, A. W., Photogrammetry applied to wind tunnel testing, AIAA J. Vol. 38, No. 6, 2000, pp. 964-971. 


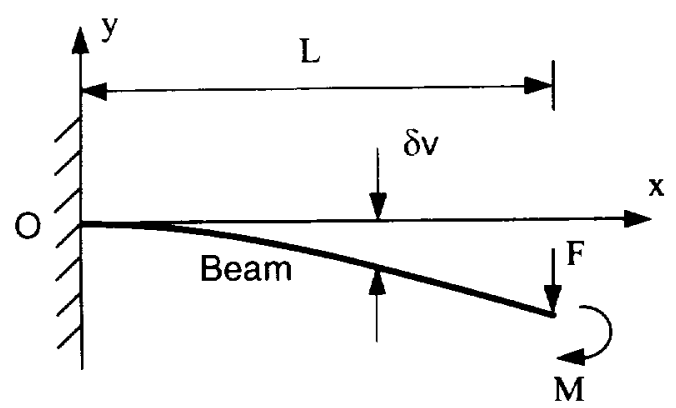

Fig. 1. A cantilever heam deformed by a force $F$ and a moment $M$.

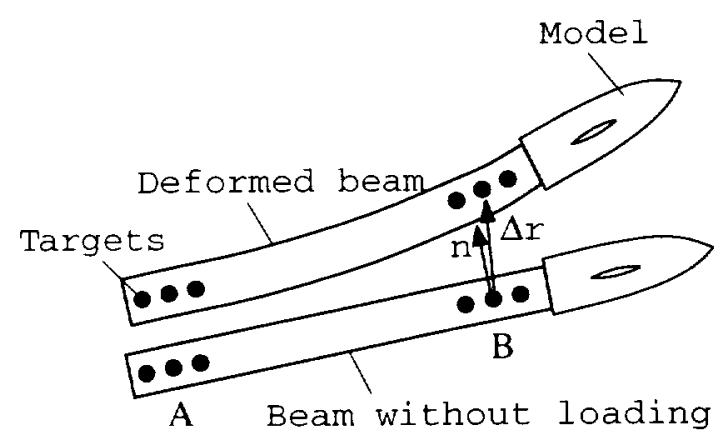

Fig. 2. Deformed sting with targets.

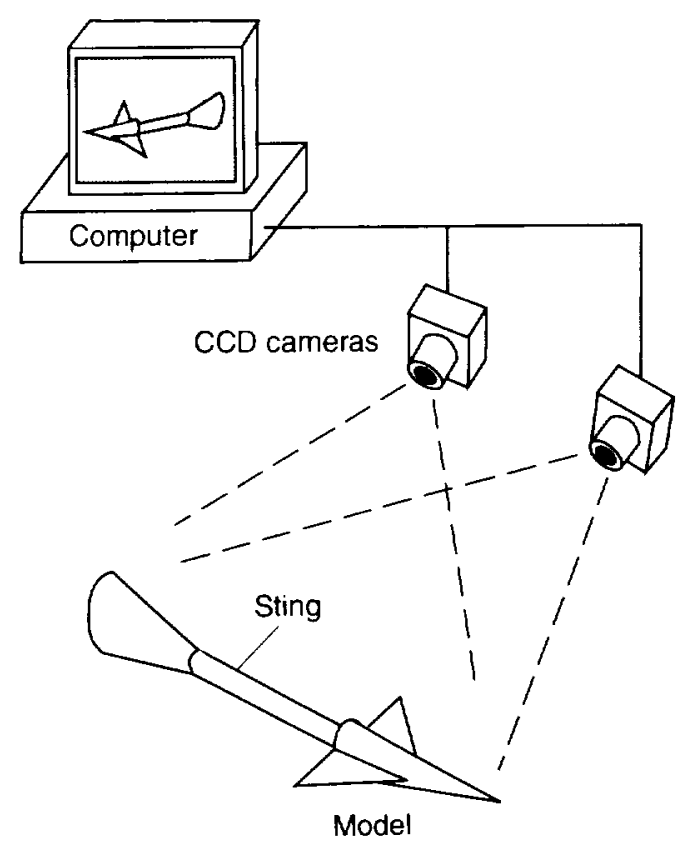

Fig. 3. Two-camera videogrammetric system for deformation measurements in wind tunnels.

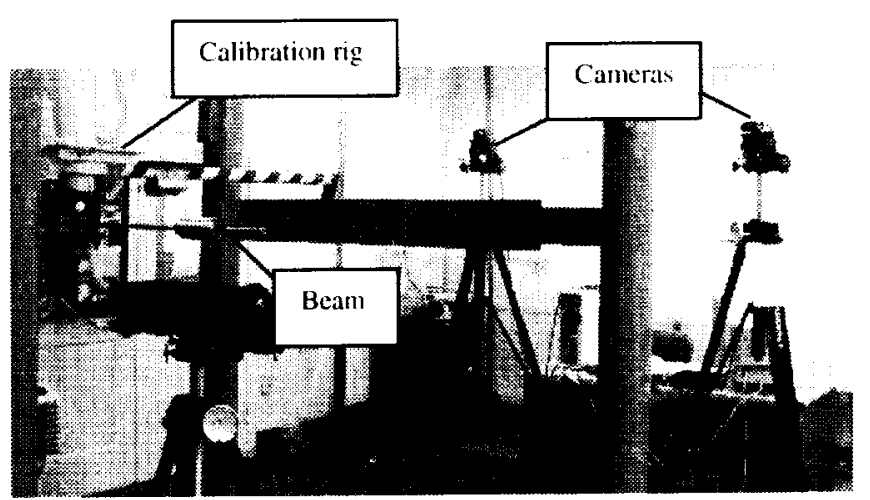

Fig. 4. Calibration facility and videogrammetric system.

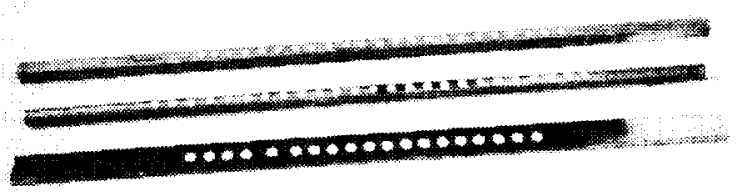

Fig. 5. Three simple beams with targets.

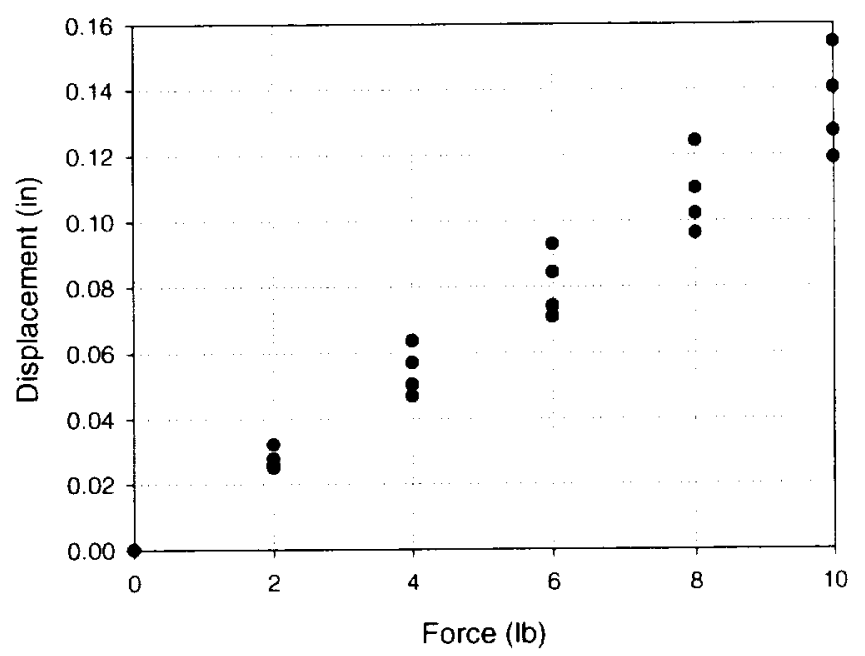

Fig. 6. Local displacement as a function of the normal force at four loading positions for a brass beam. 


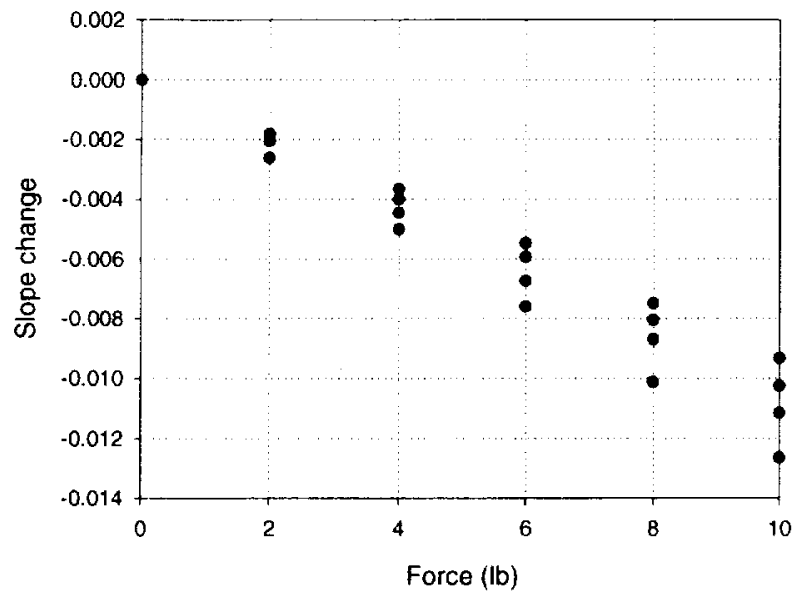

Fig. 7. Local slope change as a function of the normal force at four loading positions for a brass beam.

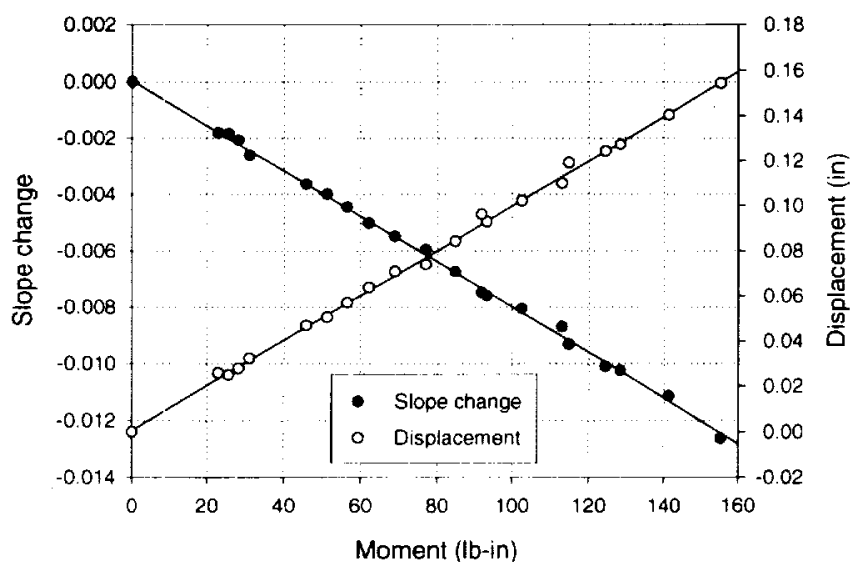

Fig. 8. Local displacement and slope change as a function of the pitching moment for a brass beam.

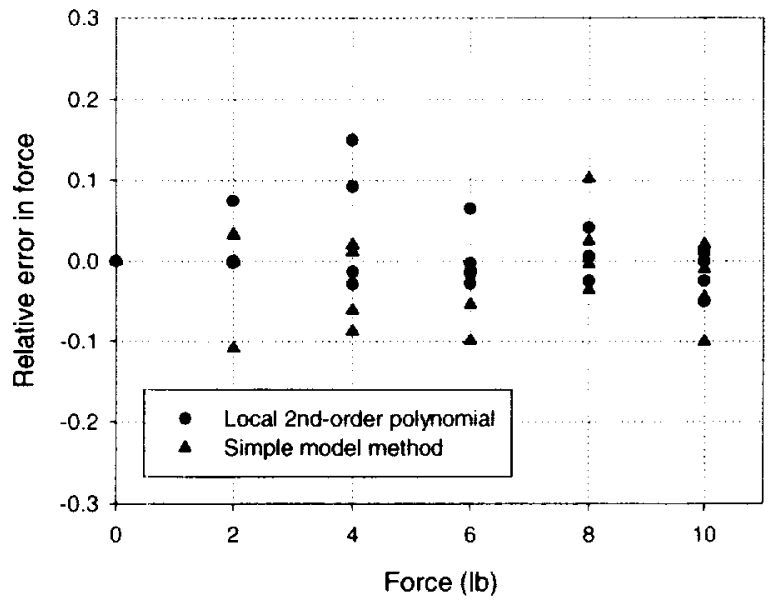

(a) (see caption in Fig. 9)

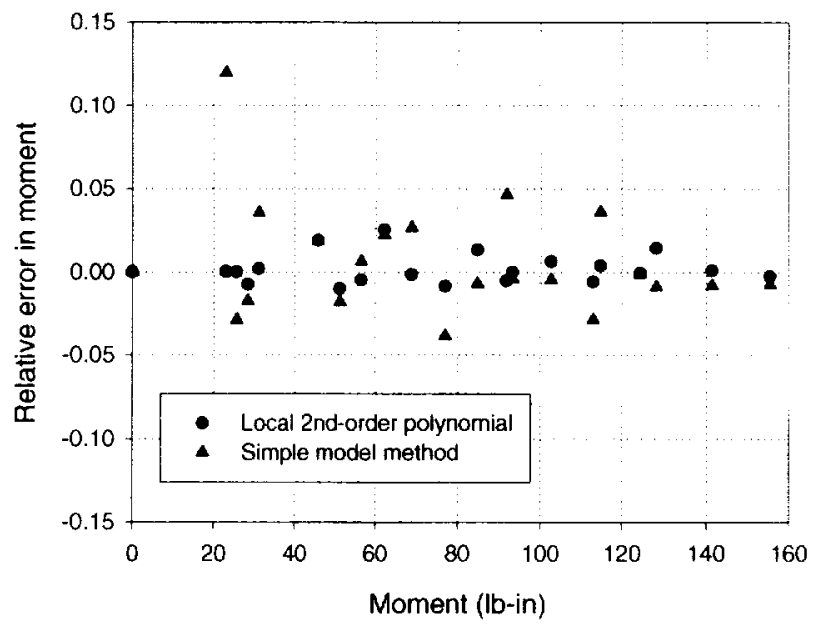

(b)

Fig. 9. Relative errors in (a) the normal force and (b) pitching moment for a brass beam when the data reduction method is based on local displacement and slope change.

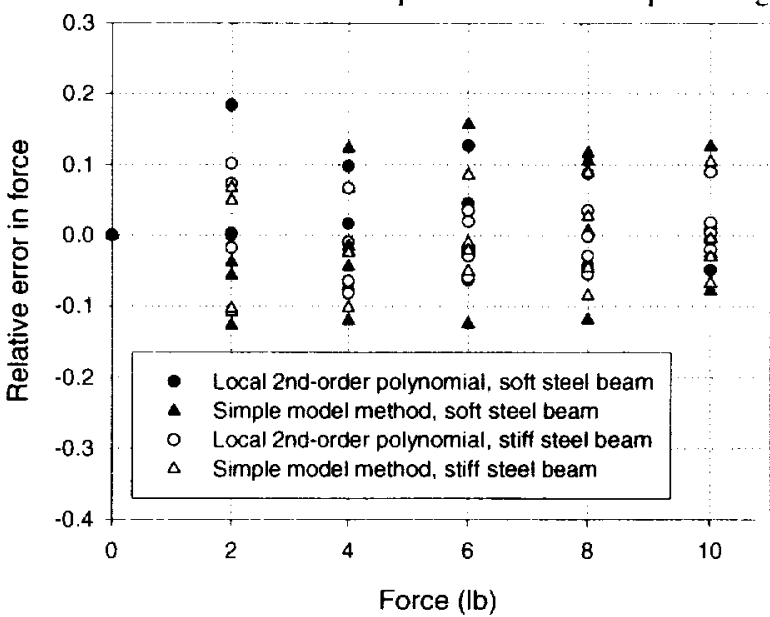

(a) (see caption in Fig. 10)

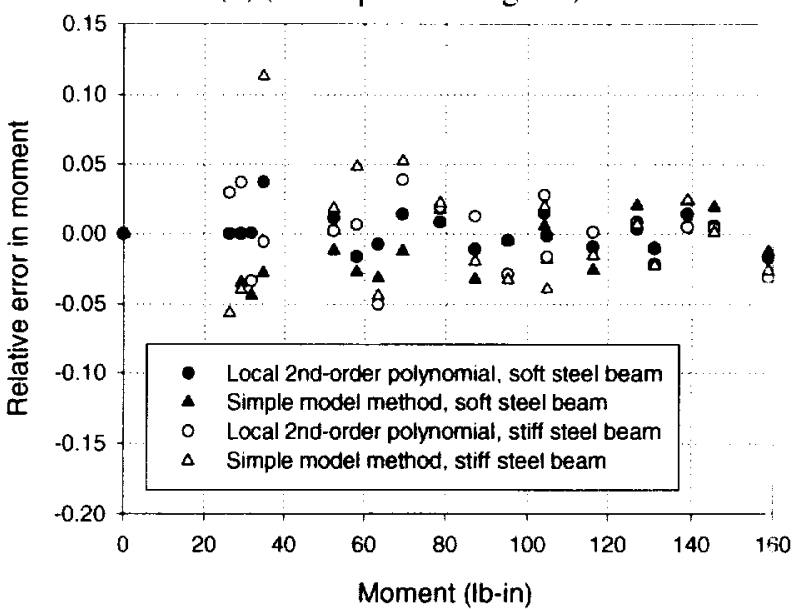

(b)

Fig. 10. Relative errors in (a) the normal force and (b) pitching moment for a soft steel beam and a stiff steel beam when the data-reduction method is based on the local displacement and slope change. 


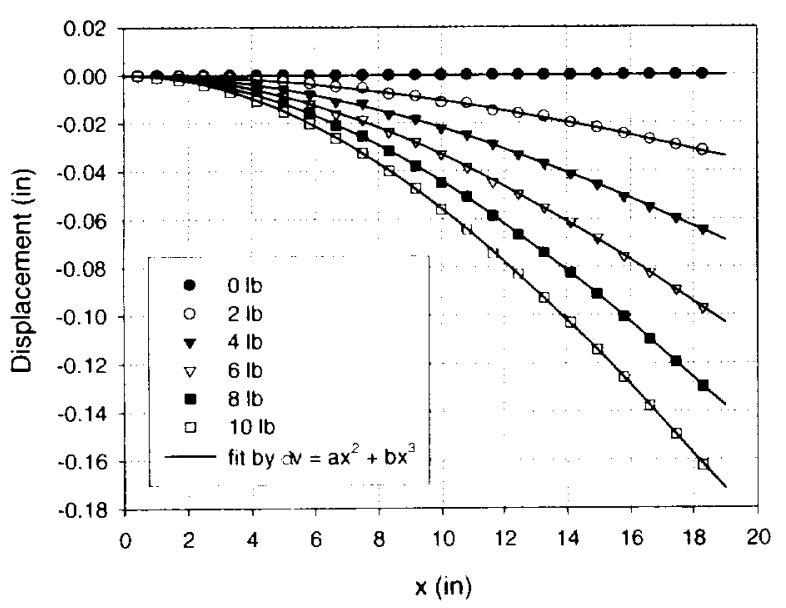

Fig. 11. The measured deformation profiles of a brass beam and the fit by $\delta v(x)=a x^{2}+b x^{3}$ at the loading position $L$

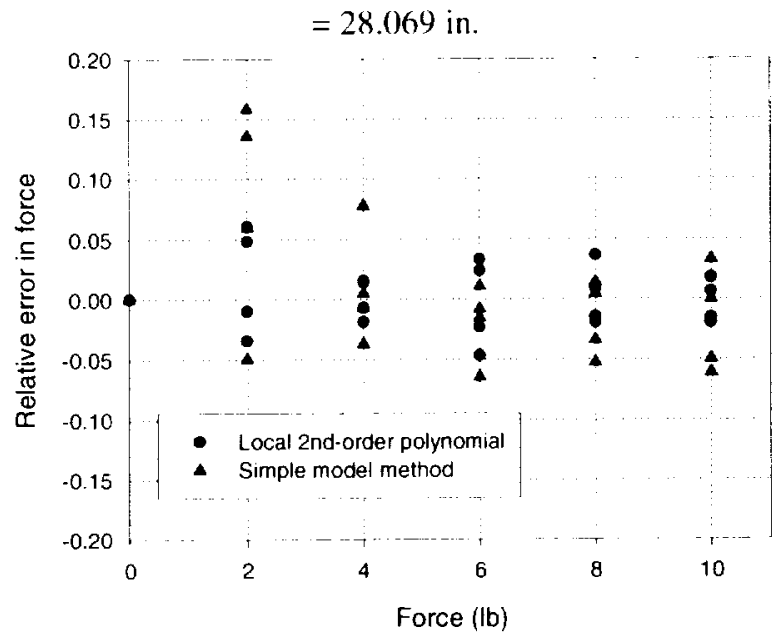

(a) (sec caption in Fig. 12)

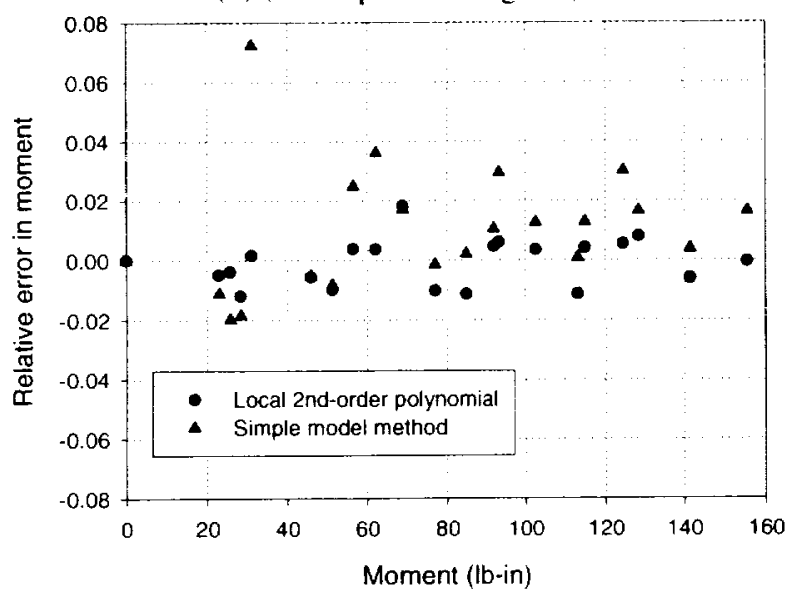

(b)

Fig. 12. Relative errors in (a) the normal force and (b) pitching moment for a brass beam when the data-reduction method is based on the global deformation profile.

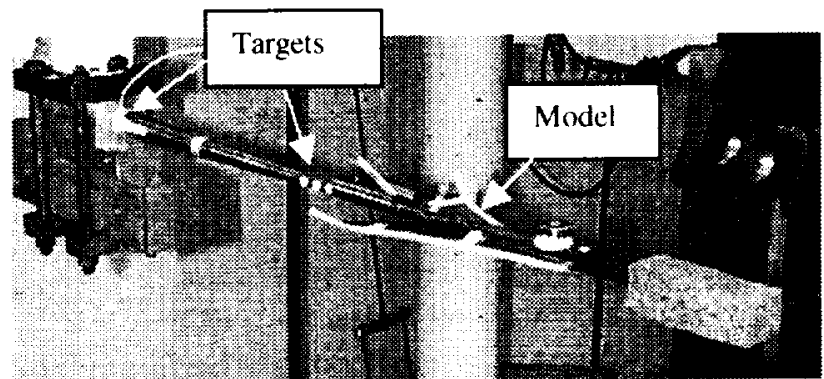

Fig. 13. The sting-model configuration with targets,

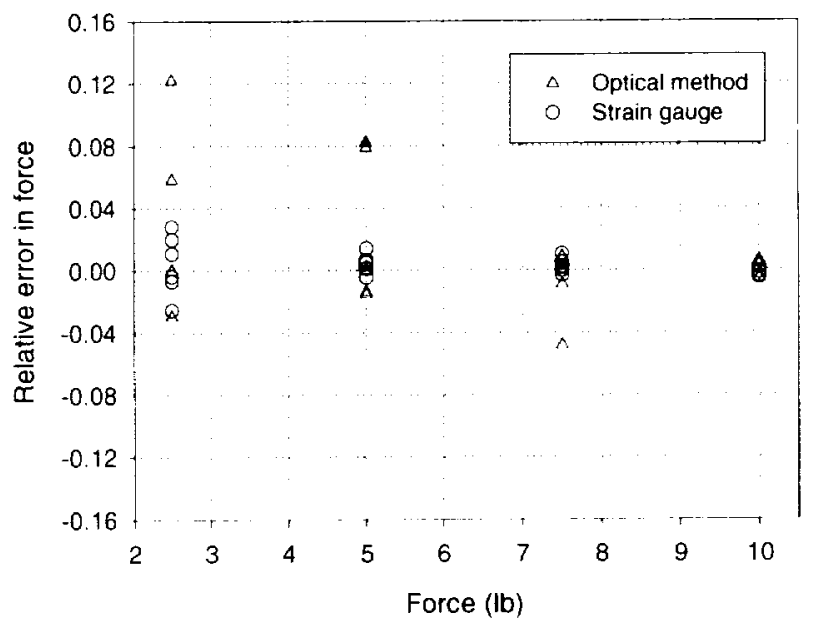

(a) (see caption in Fig. 14)

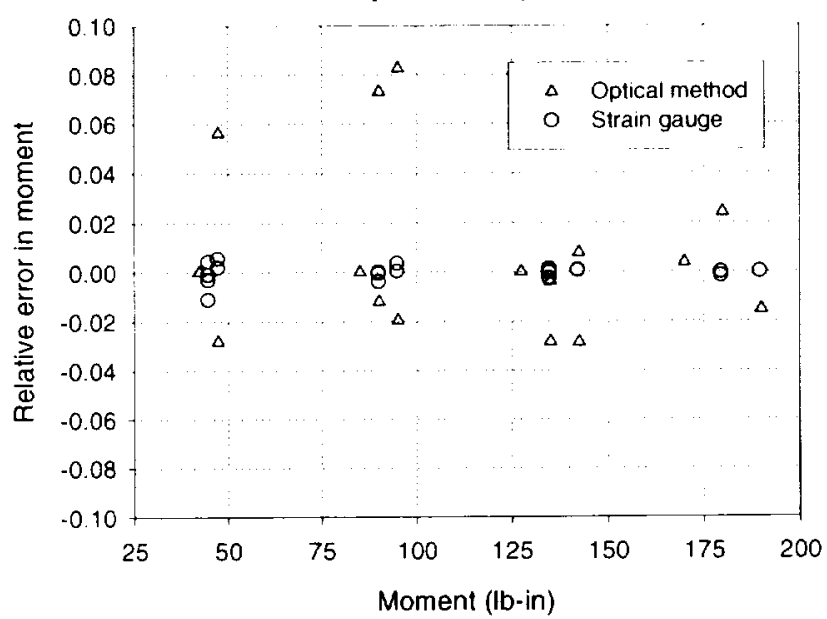

(b)

Fig. 14. Comparison between the optical method and strain gauges in measurements of (a) the normal force and (b) pitching moment for the steel sting-model configuration. The optical method is based on the local displacement and slope change. 


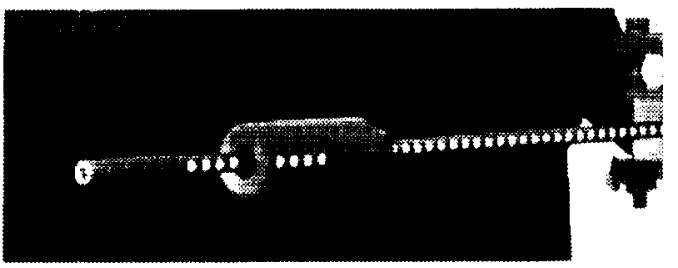

Fig. 15. Three-force beam with targets.

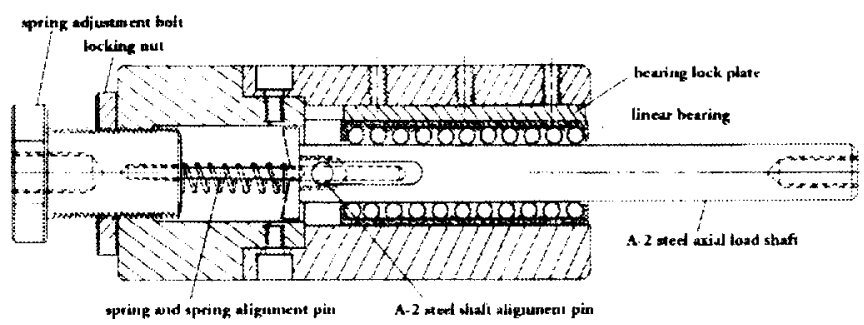

Fig. 16. Structure of the spring/bearing device.

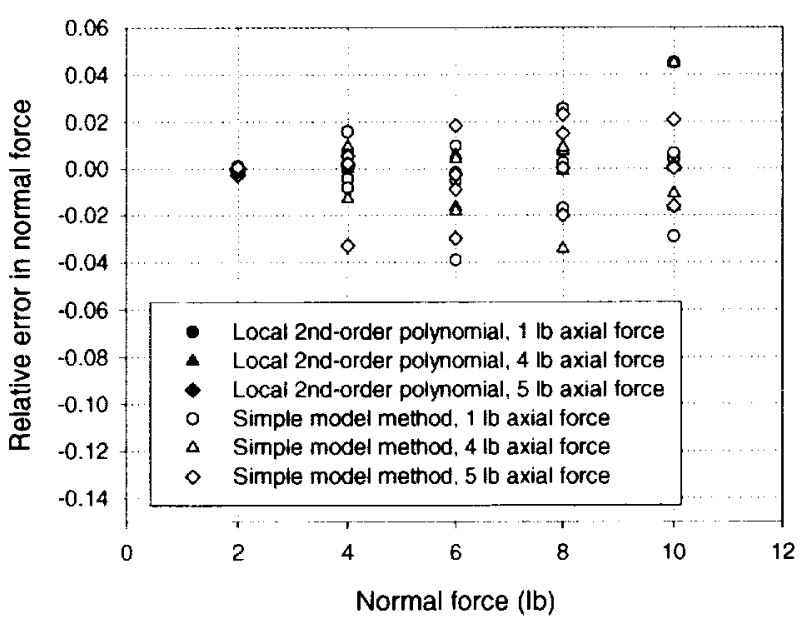

(a)

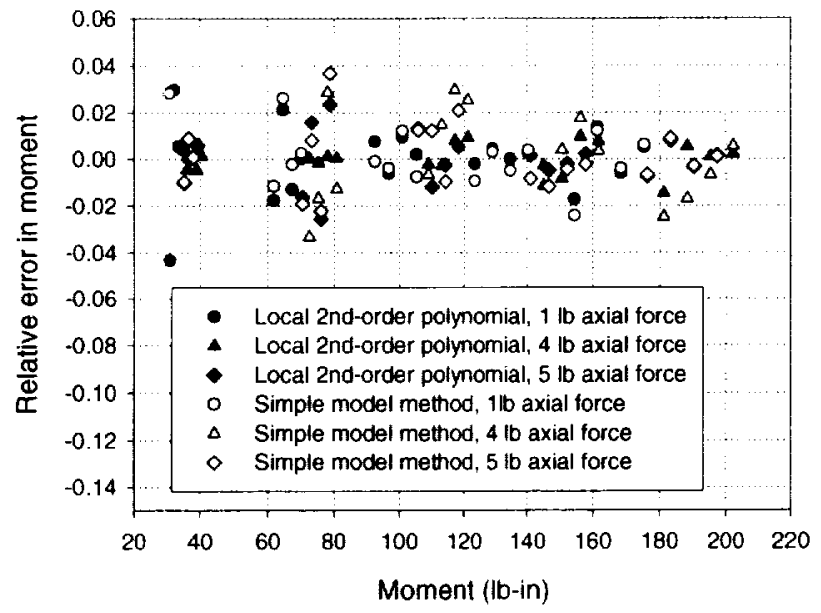

(b)

Fig. 17. Relative errors in (a) the normal force and (b) pitching moment for the three-force beam at different axial loads. The data-reduction method is based on the local displacement and slope change.

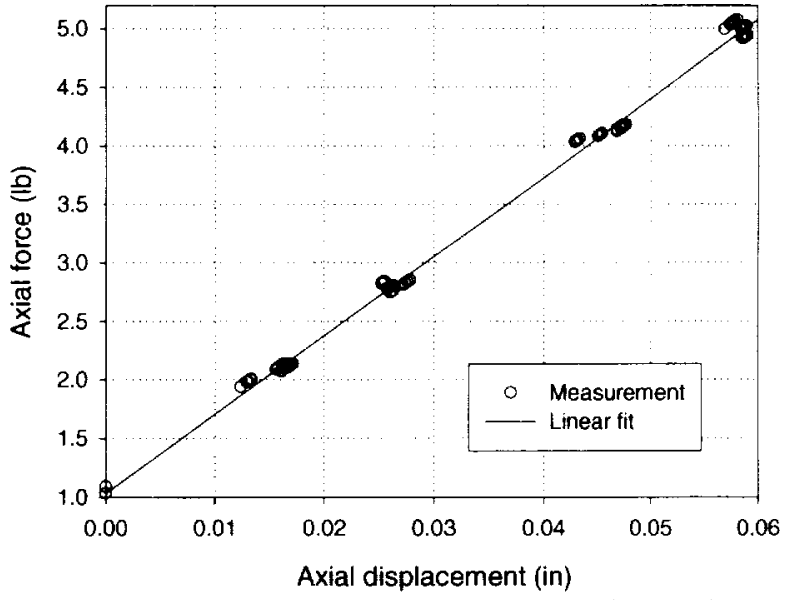

Fig. 18. The axial force as a function of the axial displacement.

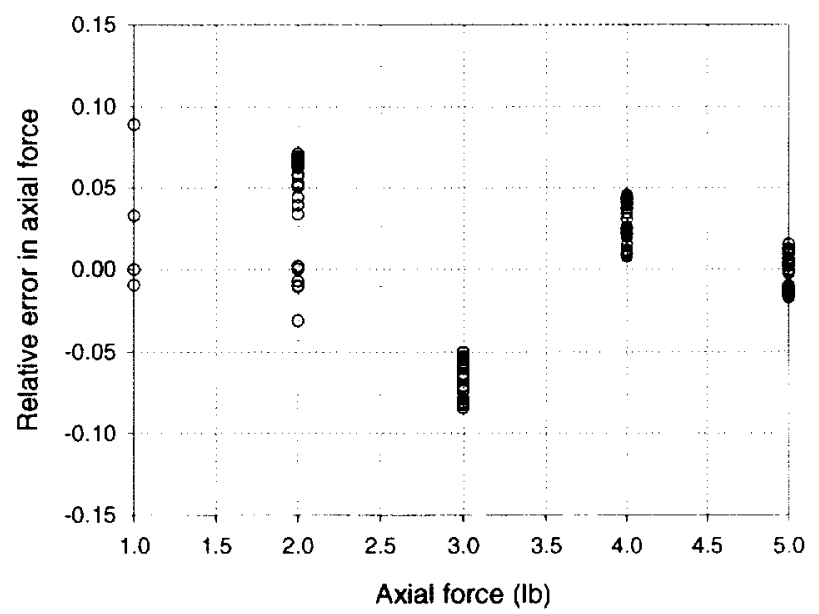

Fig. 19. Measurement errors in the axial force for the threeforce beam at different normal forces and loading positions. 